\title{
Effect of particle size and interparticle force on the fluidization behavior of gas-fluidized beds
}

\author{
J. M. Valverde, ${ }^{1}$ A. Castellanos, ${ }^{1}$ P. Mills, ${ }^{2}$ and M. A. S. Quintanilla ${ }^{1}$ \\ ${ }^{1}$ Departamento de Electronica y Electromagnetismo, Universidad de Sevilla, Avenida Reina Mercedes s/n, 41012 Sevilla, Spain \\ ${ }^{2}$ LPMDI, Université de Marne-la-Vallée, 5 boulevard DesCartes, Champs-sur-Marne, F-77454 Marne-la-Vallée Cedex 2, France
}

(Received 14 November 2002; published 23 May 2003)

\begin{abstract}
Gas-fluidized powders of fine particles display a fluidlike regime in which the bed does not have a yield strength, it expands uniformly as the gas velocity is increased and macroscopic bubbles are absent. In this paper we test the extension of this fluidlike regime as a function of particle size and interparticle attractive force. Our results show that for sufficiently large particles, bubbling initiates just after the solidlike fluidized regime as it is obtained experimentally by other workers. A scaling behavior of the solid-phase pressure in the fluidlike regime and a predictive criterion for the onset of macroscopic bubbling are analyzed in the light of these results.
\end{abstract}

DOI: 10.1103/PhysRevE.67.051305

PACS number(s): 45.70.-n, 47.55.Kf, 47.55.Mh

\section{INTRODUCTION}

The behavior of fine powders is a keystone of an increasing number of high-technology industrial processes. For example, fluidized beds, consisting of a vertical column vessel partially filled with a powder resting on a porous filter and a flowing fluid pumped from below, are extensively used in applications that take advantage of their excellent fluid-solid mixing and transport capabilities.

Back in 1973 Geldart [1] proposed an empirical classification of powders according to their fluidization properties that has been widely used by chemical engineers. Geldart summarized his observations in terms of the particle size versus the relative density difference between the fluid phase and the solid particles. According to Geldart, Group A (aeratable) powders give a region of "homogeneous" fluidization in which the particles are supported by the drag force of the fluid and the bed expands smoothly as the fluid velocity is increased, taking the appearance of a low viscosity fluid. Above a certain fluid velocity this fluidlike regime is followed by a bubbling regime in which a large part of the gas is trapped from the dense phase by rising macroscopic bubbles and bed expansion is curtailed. More common Group $B$ powders consist of coarser and denser particles than Group $A$ powder particles and give only bubbling fluidization. This group includes sandlike materials. Group $C$ powders are very fine, cohesive powders that are incapable of fluidize and tend to rise as a slug of solids or to form channels through which the fluid will escape rather than being distributed through the bulk. The border between Group $A$ and $B$ powders is not clear cut. For instance, it has been found that the fluid pressure influences the bubbling behavior. Elevated pressure produce more homogeneous gas-solid flow structure by intensifying particle-fluid interaction and suppressing particle-particle interactions, thereby extending the uniform fluidization regime [2].

A fine powder showing an amazing behavior and used in the xerographic industry is toner. In the last decades image quality requirements leaded to a reduction of toner particle size in expenses of loosing the good flowability exhibited by the old-fashion toners, for which the interparticle attractive forces were comparable with the inertial forces. Nowadays xerographic toners are made of polymer particles with a volume average size $d_{p}<\sim 10 \mu \mathrm{m}$. For such a small particle size we would predict Group $C$ typical sticky behavior since interparticle attractive forces are several orders of magnitude larger than particle weight. The need of reducing cohesiveness was approached by means of the use of additives. The addition of fumed silica nanoparticles, which became dispersed on the surface of the toner particles, decreases the interparticle adhesion and thus causes a shift to well-behaved Group $A$ powders. Moreover, the small particle size and its low density $\left(\rho_{p} \sim 1 \mathrm{gr} / \mathrm{cm}^{3}\right)$ allowed for an extended interval of "homogeneous" fluidization in which the bed could expand uniformly up to reach solid volume fractions as low as 0.1 [3]. This behavior contrasts with the behavior of Group $A$ powders usually encountered in commercial gas-fluidized systems, for which the interval of nonbubbling fluidization is rather short.

Experiments reported by Rietema and co-workers [4], Tsinontides and Jackson [5], and Menon and Durian [6] on gasfluidized particles of size $d_{p} \geqslant 50 \mu \mathrm{m}$ indicated the absence of grain dynamics in the uniformly fluidized state. These researchers asserted that in the fluidized state the particles are static in direct contact with each other, thus providing an elastic modulus to the bed that stabilizes it against small perturbations. This picture was indeed upheld by theoretical investigations based on the kinetic theory. Koch and Sangani [7] derived the particle-phase pressure of a homogeneous suspension of particles in a gas interacting via instantaneous hard-sphere collisions and hydrodynamic interactions. Their linear stability analysis indicated that the homogeneous state of a fluidlike gas-fluidized bed is always unstable, leading them to the conclusion that bubbling can be only restrained by a solidlike behavior of the expanded beds in agreement with Rietema and other's experiments. However, it contradicted empirical observations [8] on some particular gasfluidized systems belonging to Group $A$ in the Geldart's classification scheme that exhibit a fluidlike "homogeneous" regime. Our measurements on the yield strength and diffusivity of our gas-fluidized fine powders with reduced cohesiveness clearly showed that both regimes, the solidlike (below a certain gas velocity $v_{c}$ larger than the minimum fluidization velocity $v_{m f}$ ) and the fluidlike (above $v_{c}$ ), might be encountered in the so-called interval of "homogeneous" fluidization [9]. 
A deeper insight into the local dynamics of the fluidlike regime has recently revealed the presence of mesoscale spatiotemporal structures and short-lived voidage instabilities [3] whose characteristic temporal frequency gradually increases with gas velocity. Well-organized fluctuations $[10,11]$ and transient voids [12] have also been discovered in liquidfluidized beds. Duru and Guazelli showed that in liquid fluidization local voids grew into macroscopic real bubble-like structures for sufficiently high density beads (particle density/liquid density $\rho_{p} / \rho_{f} \approx 8$ ) [12]. Thus, in close similarity with observations in liquid-fluidized beds, we should conclude that our gas-fluidized beds are actually unstable throughout the fluidlike nonbubbling regime. Even though this instability does not grow into fully developed bubbles, it certainly denies the applicability of hydrodynamic linear or weakly nonlinear stability analyses that have been employed in the past to predict the onset of bubbling [13]. Still the question of why large bubbles develop above certain fluid velocities remains unexplained.

Undoubtedly bubbling must be inhibited by some complex hydrodynamic mechanism for sufficiently small particles, yet a relative role of interparticle forces should not be unequivocally dismissed. Numerical simulations of bubbling beds have recently shown that when the attractive interparticle force was increased the average size of bubbles decreased and the coalescence rate of bubbles slowed down [14]. It is well known that van der Waals attractive force, much larger than particle weight for micrometer-sized particles, causes the aggregation of fluidized fine particles [15]. Independent empirical observations suggest that the size of aggregates could influence the transition to the bubbly regime: The homogeneity of fluidization has been substantially improved in some cases when the aggregates could be disrupted by means of external agents such as vibration [16] or ultrasonics [17]. Changes occurring during fluidization of some fcc catalysts have been observed as the interparticle attractive forces were increased by increasing temperature [18]. Bubbling of beds of metallic particles could be suppressed controlling interparticle forces by means of an external magnetic field [19].

One of our aims in this paper is to contribute to fill the gap between the observations on one side of a bubble-free fluidlike regime for small particles and on the other side of just bubbling fluidization for larger particles. Following a suggestion by Jackson [20], the extension of the fluidlike regime interval and the onset of bubbling are experimentally investigated using specially designed powders of several particle sizes and different cohesiveness. This work will allow us to elucidate a role of interparticle forces and particle size on fluidization behavior.

\section{EXPERIMENTAL POWDERS}

Toner particles made of the random copolymer styrene $n$-butylmethacrylate $\left(\rho_{p}=1.14 \mathrm{~g} / \mathrm{cm}^{3}\right)$ were produced by an attrition process followed by size classification by means of a cyclone separator apparatus. In this way we had available four sets of powders with volume averaged particle size $d_{p}$ $=19.1,15.4,11.8$, and $7.8 \mu \mathrm{m}$. These powders were subse-
TABLE I. $d_{p}$, particle size; $N$, average number of particles per aggregate in the fluidized bed; $k$, average ratio of aggregate size to particle size; $\phi_{b}^{\text {expt }}$, experimental value of the solid volume fraction at the initiation of bubbling; $\phi_{b}^{\text {theor }}$, theoretical value of the solid volume fraction at the initiation of bubbling according to Eq. (7); Fr, Froude number at the bubbling point based on aggregate size (values corresponding to $32 \%$ SAC).

\begin{tabular}{lccccc}
\hline \hline$d_{p}(\mu \mathrm{m})$ & $N$ & $k$ & $\phi_{b}^{\text {expt }}$ & $\phi_{b}^{\text {theor }}$ & Fr \\
\hline 7.8 & 63 & 5.22 & 0.089 & 0.087 & 0.14 \\
11.8 & 23.7 & 3.55 & 0.140 & 0.146 & 0.09 \\
15.4 & 12.4 & 2.72 & 0.177 & 0.188 & 0.07 \\
19.1 & 9.6 & 2.45 & 0.228 & 0.229 & 0.04 \\
\hline \hline
\end{tabular}

quently blended with nanoparticles of fumed silica, which became dispersed on the toner particle surface. $32 \%$ and $8 \%$ of surface area coverage (SAC) were used. The addition of these nanoparticles allowed for a reduction of the interparticle adhesion force because silica is considerably harder than polymer and thus the additive increases the contact hardness; silica also decreases the adhesion force by reducing the typical size of the surface asperities at contact $[21,22]$. In the fluidlike regime toner particles are aggregated. Assuming that in sedimentation aggregates behave like effective spheres with a hydrodynamic radius equal to their radius of gyration $R$, we derived elsewhere the average number of particles per aggregate $(N)$ and the average ratio of aggregate size to particle size $\left(k \equiv 2 R / d_{p}\right)[15,23]$ (see Table I).

\section{EXPERIMENTAL SETUP}

In the experiments reported in this paper a powder sample of mass $m$ was held in a $r=2.21 \mathrm{~cm}$ radius polycarbonate cylinder, the base of which is a sintered metal filter of $5 \mu \mathrm{m}$ pore size. For this bed radius wall effects are negligible [24]. A dry nitrogen tank furnished with a mass flow controller supplied adjustable gas flow. All the measurements started by driving the powder into the bubbling regime by imposing a very large gas flow to the bed. Once the powder is in the bubbling regime it had lost memory of its previous history. Then the gas flow was decreased down to a given value. After the bed had reached a stationary state its height $h$ was read from an ultrasonic sensor and the average solid volume fraction $\phi\left[\phi=m /\left(\rho_{p} \pi r^{2} h\right)\right]$ was computed.

\section{RESULTS}

In Fig. 1 the solid volume fraction is plotted as a function of the superficial gas velocity $v_{g}$ for powders with the same SAC (32\%), i.e., same value of interparticle van der Waals force $\left(F_{v d W} \simeq 1.6 \mathrm{nN}[15]\right)$. In Fig. 1 we delineate the transitions between the different fluidization regimes. The gas velocity at the transition to the solidlike regime $v_{c}$ is taken from the point at which the development of enduring contacts cause a slow down of the settling process [15]. We must note, however, that the transition solidlike-fluidlike takes place along an interval of gas velocities in which transient 


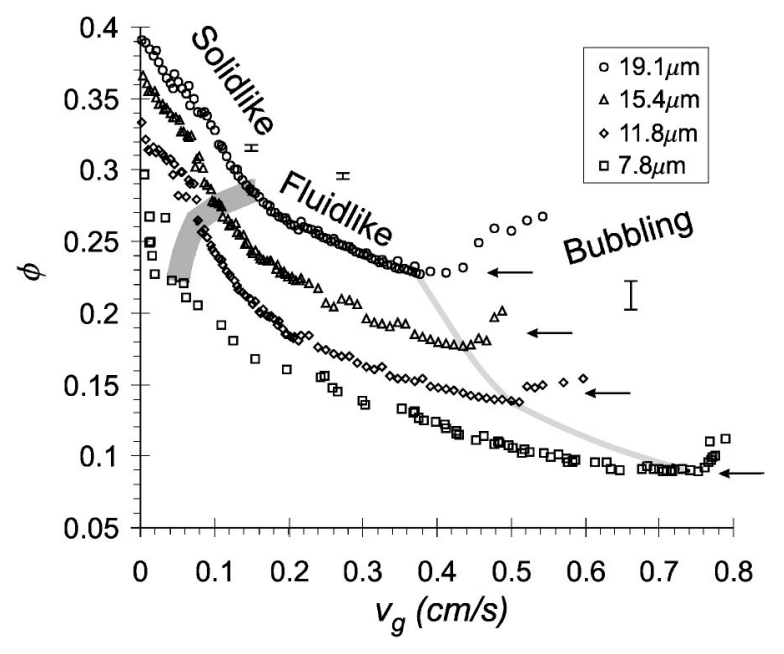

FIG. 1. Average solid volume fraction $\phi$ of the fluidized powder as a function of the superficial gas velocity $v_{g}$. Particle size is indicated in the inset. Surface additive coverage is held constant $(32 \%)$. The limits between the solidlike, fluidlike, and bubbling regimes are shown. Error bars represent the typical standard deviation of the time signal of $\phi$ for a given $v_{g}$ in each regime. The arrows show the predicted values for the solid volume fraction at incipient bubbling, using a modified version of the FG criterion.

fluidlike regions alternate with transient solid networks. This issue is extensively discussed elsewhere [3]. In the fluidlike regime $\phi$ decreases steadily as the gas velocity is increased down to the minimum value $\phi_{b}$ that the two-phase system is able to sustain. If the gas velocity is further increased above this critical point, macroscopic bubbles rise across the bed and $\phi$ increases, with oscillations of the free surface due to gas bubbles burst. The gas velocity at incipient macroscopic bubbling $v_{b}$ is well determined and we take it from the point in Fig. 1 at which the solid fraction starts to increase as the gas velocity is increased.

\section{DISCUSSION}

A clear outcome from Fig. 1 is that the fluidlike regime shortens as particle size is increased. Let us consider the interval $\Delta v_{g} \equiv v_{b}-v_{c}$, which is plotted in Fig. 2 against the ratio of interparticle van der Waals force $F_{v d W}$ to particle weight $W_{p}$ (granular Bond number $\mathrm{Bo}_{g} \equiv F_{v d W} / W_{p}$ ). It is seen that $\Delta v_{g}$ shows a logarithmic increase with $\mathrm{Bo}_{g}$. An extrapolation of the results gives $\Delta v_{g} \rightarrow 0$ for $\mathrm{Bo}_{g}<\sim 10$, indicating that the fluidlike regime should shrink to zero for fluidized beds of large enough particles. For instance, in the case of glass beads $\left(\rho_{p} \simeq 2.5 \mathrm{~g} / \mathrm{cm}^{3}\right)$ we estimate $F_{v d W}$ $\equiv A d_{a} /\left(24 z_{0}^{2}\right) \simeq 8 \mathrm{nN}$ (Hamaker constant $A \simeq 1.5 \times 10^{-19} \mathrm{~J}$, typical asperity size $d_{a} \simeq 0.2 \mu \mathrm{m}$ [25], and minimum interparticle distance $z_{0} \simeq 4 \AA$ [26]), and thus the condition $B o_{g}$ $<\sim 10$ would be met for $d_{p}>\sim 40 \mu \mathrm{m}$. Thus, for particles of size larger than $\sim 40 \mu \mathrm{m}$, macroscopic bubbling would initiate immediately after the solidlike regime. This is in accordance with experiments reported in the literature [6] and with a fluidization test we made in our lab, using a system of glass beads with $d_{p} \simeq 60 \mu \mathrm{m}$. Interestingly, a similar threshold is found for the aggregation of particles $[15,27]$, suggest-

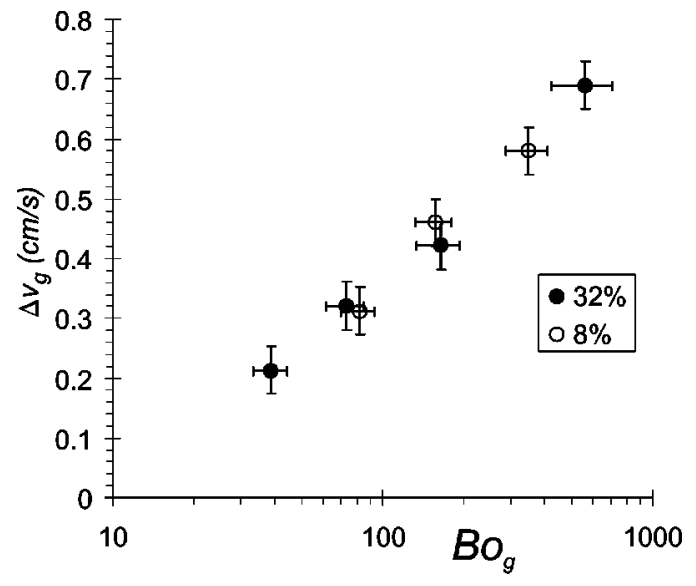

FIG. 2. Extension of the fluidlike regime as a function of the ratio of interparticle van der Waals force to particle weight. The $\mathrm{SAC} \%$ of the toner particles is indicated.

ing that the formation of low-density aggregates plays a crucial role in promoting the gas-solid interaction and thus in preventing the growth of large bubbles along an extended pseudoturbulent fluidlike interval.

Figure 3 shows the effect of reducing the surface silica coverage from $32 \%$ down to $8 \%$ (thus increasing the van der Waals force from $1.6 \mathrm{nN}$ up to $3.4 \mathrm{nN}$ [15]) for a given particle size (the same effect is obtained for other particle sizes). We observe that, in the fluidlike regime, the bed of more cohesive particles shows larger values of $\phi$ for a given gas velocity, whereas the opposite happens in the solidlike regime. This behavior may be rationalized as follows: As the additive surface coverage is decreased the interparticle attractive force increases and particles tend to be slightly more aggregated [15]. Thus for larger aggregates the gas velocity required to reach a given value of the solid volume fraction

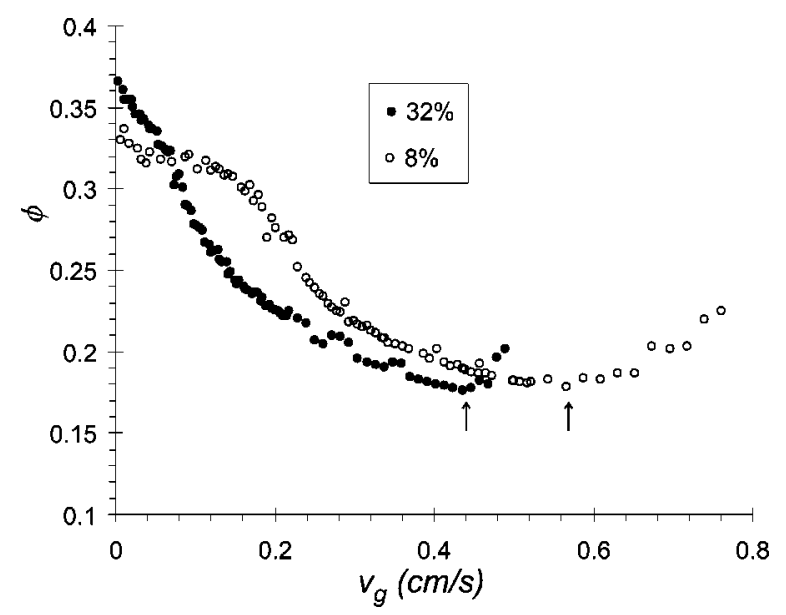

FIG. 3. Average solid volume fraction $\phi$ of the fluidized powder as a function of the superficial gas velocity $v_{g}$ for powders with the same particle size $(15.4 \mu \mathrm{m})$ but different SAC (shown). The arrows indicate the bubbling point. This figure has been recently reviewed by Sundaresan [39]. Please note that the figure shown in that review displays the interstitial gas velocity $v_{i}$ instead of the superficial gas velocity $v_{g}\left[v_{i}=v_{g} /(1-\phi)\right]$ for the powder with $32 \%$ SAC. This minor difference does not alter the discussion presented in the text. 
in the diluted fluidlike regime should be higher as we see in Fig. 3. Accordingly the gas velocity needed to reach the bubbling regime increases with the reduction of surface additive. On the other side when the gas velocity is decreased down to the point at which the solidlike regime is reached aggregates roll or slide over each other until they land at static positions and form permanent networks. The higher the interparticle attractive force the less efficient is the rearrangement of aggregates leaving behind larger void spaces. This leads to smaller values of $\phi$ for a given gas velocity as we see in Fig. 3. We have plotted in Fig. 2 the extension of the fluidlike regime interval for the beds with increased cohesiveness and it can be seen that $v_{b}-v_{c}$ follows the same law with $\mathrm{Bo}_{\mathrm{g}}$.

Even though our experiments are restricted to toner particles for which the van der Waals force provides the main source of interparticle attraction, recent numerical simulations as well as experiments with magnetic and wet particles also show that the role of interparticle force on fluidization behavior must scale with the particle weight [28]. Furthermore, our results agree qualitatively with the results of Rhodes et al. [28] who obtain by means of a Discrete Element Method simulation that the interval of nonbubbling bed expansion shrinks to zero for $\mathrm{Bo}_{g} \rightarrow 1$.

\section{A PREDICTIVE CRITERION FOR THE INITIATION OF MACROSCOPIC BUBBLING}

More than half a century ago Wilhelm and Kwauk delineated the transition to the bubbling regime based on the empirical Froude number criterion, $\mathrm{Fr}=v^{2} /\left(g d_{p}\right)>1$ where $v$ is the fluid velocity and $g$ is the gravity field [29]. However, researchers found an order of magnitude of difference between, for instance, air-fluidized glass beads $(\mathrm{Fr}=1.1)$ and air-fluidized lead shot $(\mathrm{Fr}=85)$ [30]. In addition, the criterion failed to predict the observed instability in liquid-fluidized beds, for which $\mathrm{Fr} \sim 0.1<1[12,30]$. Using the effective size of the aggregates for our gas-fluidized powders we estimate Fr $\sim 10^{-1}-10^{-2}$ (Table I) at incipient bubbling, thus denying the applicability of the Wilhelm and Kwauk criterion in close analogy with liquid-fluidized beds. Later in 1984, Foscolo and Gibilaro (FG) [31] formulated the predictive criterion for $\phi_{b}$,

$$
\left(g d_{p}\right)^{1 / 2}=0.56 n v_{t} \phi_{b}^{1 / 2}\left(1-\phi_{b}\right)^{n-1} .
$$

Here $v_{t}$ is the Stokes settling velocity of a single particle and $n$ is the exponent in the phenomenological RichardsonZaki (RZ) law [32], $v_{g}=v_{t}(1-\phi)^{n}$. By including the prefactor 0.56 in the right hand side of the equation FG admitted $n=4.8$ according to earlier RZ empirical observations on the expansion and settling of fluidized beds at low particle Reynolds numbers [32]. We find in the literature a variation in the empirical values of the Richardson-Zaki exponent maybe due to wall effects, residual polydispersity effects and inertial screening effects as pointed out by Snabre and Mills [33]. As we are dealing with gas-fluidized beds we give above a simplified version of the FG criterion by neglecting the ratio of gas density to particle density.
The original derivation of Eq. (1) was based on the general criterion proposed by Wallis to describe bubbles as concentration shocks that originate when the propagation velocity of a voidage disturbance $\left(u_{\phi}\right)$ rises faster than the elastic response rate of the bed. FG used for $u_{\phi}$ the well-established relation

$$
u_{\phi}=-\phi \frac{d v_{g}}{d \phi}
$$

where $v_{g}$ was related to $\phi$ by the RZ law. On the other hand, the elastic wave velocity $u_{e}$ must be calculated as

$$
u_{e}=\left(\frac{1}{\rho_{p}} \frac{\partial p}{\partial \phi}\right)^{1 / 2}
$$

where $p$ is the particle-phase pressure. As earlier suggested by Verloop and Heertjes [34], Foscolo and Gibilaro neglected particle inertia and related $p$ to the fluid-particle drag force in an ideal homogeneous state, arriving at

$$
p=\frac{1}{3} n \rho_{p} g d_{p} \phi^{2} \sim \rho_{p} g d_{p} \phi^{2} .
$$

This is a wrong derivation, however, since the pressure variation cannot be due only to a dissipative process but, as noted by Batchelor [13] and Jackson [35], momentum transport by particle fluctuations and collisions must be necessarily considered. The linear stability analysis of Batchelor [13] yields for the particle pressure $p \sim \rho_{p} v_{g}^{2} \phi F(\phi)$, where $F(\phi)$ is some function of the solids fraction. Taking into account that when a particle is effectively isolated $(\phi \rightarrow 0) p \rightarrow 0$ and that $p$ must be zero again when the particles are locked in the packed assembly $\left(\phi \rightarrow \phi_{c}\right)$, Batchelor suggested the simple representation $F(\phi) \approx\left(\phi / \phi_{c}\right)\left[1-\left(\phi / \phi_{c}\right)\right]$. This rough estimate drove him to values of $p$ in the dilute limit comparable to the FG prediction [36]. Still there is not a general consensus on a correct formulation of the particle pressure.

It must be remarked that, even though the bed manifests a smooth appearance to the naked eye in the fluidlike regime, the existence of mesoscale spatiotemporal structures [3,12] indicates that the fluidlike regime is not truly homogeneous after all. In fact, as pointed out by Jackson [35], the FG criterion fails to predict the onset of primary instability observed in liquid fluidized beds consisting of voidage waves that do not develop into recognizable bubbles. In spite of these severe drawbacks, the survival of the FG criterion to discriminate visible bubbling along years of experiments is fascinating. In their paper Foscolo and Gibilaro reported good agreement with experimental data on liquid and gas fluidized beds at both laminar and turbulent conditions [31]. Over the next years the FG criterion was successfully applied under systematic variations of relevant parameters on the bubbling point of gas-fluidized beds such as pressure, temperature, and addition of fines [37]. Moreover, measurements of the collisional particle pressure in liquid-solid flows yielded, in the range $\phi<\sim 0.3$, similar results to those predicted from the FG model, whereas results of collision-based models were in poor correspondence with the experiments [36]. Therefore it seems that the scaling $p \sim \rho_{p} g d_{p} \phi^{2}$ can be taken at least as an empirical fact. 
Let us extend Eq. (1) to our case of aggregated fine particles. According to our previous model [23], aggregates are viewed like effective spheres with a hydrodynamic radius equal to their radius of gyration and undergoing a collisional dynamics. The average number of aggregated particles $N$ and the ratio of aggregate size to particle size $k$ are derived fitting a generalized RZ law $v_{g}^{*}=v_{t}^{*}\left(1-\phi^{*}\right)^{n}$, where $v_{t}^{*}=v_{t} N / k$ [23] is the Stokes velocity of a single aggregate and $\phi^{*}$ $=\phi k^{3} / N[23]$ is the volume fraction filled by the aggregates, to the results of settling experiments $[15,23]$. We admit that the voidage wave velocity can be obtained as

$$
u_{\phi}^{*}=-\phi^{*} \frac{d v_{g}^{*}}{d \phi^{*}}=k^{2} \phi v_{t} n\left(1-\phi \frac{k^{3}}{N}\right)^{n-1} .
$$

Following the same approach a straightforward generalization of the particle pressure is given by $p^{*}$ $\sim \rho^{*} g k d_{p}\left(\phi^{*}\right)^{2}$, where $\rho^{*}=\rho_{p} N / k^{3}[23]$ is the density of the aggregate. Thus, for our effective system of aggregates, the elastic wave velocity can be calculated as

$$
u_{e}^{*}=\left(\frac{1}{\rho^{*}} \frac{\partial p^{*}}{\partial \phi^{*}}\right)^{1 / 2} \sim\left(g d_{p} \phi \frac{k^{4}}{N}\right)^{1 / 2} .
$$

If we admit that visible bubbling is a consequence of a nonlinear process leading to the development of concentration shocks we can recover the Wallis criterion relating bubbling to the crisis that results from the balance between the voidage and elastic waves velocities. Thus, equating $u_{\phi}^{*}$ $\sim u_{e}^{*}$ we obtain a predictive criterion for the solid volume fraction at the onset of macroscopic bubbling

$$
\left(g d_{p}\right)^{1 / 2} \sim n v_{t} N^{1 / 2} \phi_{b}^{1 / 2}\left(1-\phi_{b} \frac{k^{3}}{N}\right)^{n-1} .
$$

As can be seen in Table I this modified criterion yields values of $\phi_{b}$ quite close to the experimental ones. It must be stressed, however, that we propose an order of magnitude for $u_{e}^{*}$ based on the scaling of the particle pressure $p^{*}$ $\sim \rho^{*} g k d_{p}\left(\phi^{*}\right)^{2}$. Since aggregates of our cohesive particles have a fractal dimension $(D=\ln N / \ln k)$ close to 2.5 (diffusion-limited aggregation limit) [15] we can write $p$ $\sim \rho_{p} g d_{p} k^{3 / 2} \phi^{2}$ where the influence of interparticle forces is explicit in the ratio of aggregate size to particle size $k$, showing that the aggregation of particles contributes to rise particle pressure and thus has a stabilizing action.

\section{CONCLUSIONS}

By extending the range of particle size downwards we have illustrated the connection between the two mechanisms that have been proposed in the literature as responsible for suppressing bubbling in fluidized beds, on one hand hydrodynamic forces and on the other interparticle forces. Although both mechanisms seemed to be contradictory the present work shows that they are rather complementary. The extent of the fluidlike regime and thus the relative role of hydrodynamic stabilization shortens as particle size is increased, indicating that large bubbles are just restrained by yield stresses for sufficiently large particles. Nevertheless the inherent instability of the fluidlike regime confirms that bubble macrostructures cannot be the result of a linear departure from a homogeneous state but a consequence of a complex nonlinear hydrodynamic interaction in which interparticle forces play a relative role by inducing particle aggregation. Application of the Wallis criterion to predict the onset of bubbling suggests that the particle pressure in the pseudoturbulent fluidlike regime scales as $p \sim \rho_{p} g d_{p} k^{3 / 2} \phi^{2}$. This law is in contradiction with the sublinear dependence on $\phi$ theoretically predicted for a homogeneous state [7] but agrees with direct measurements made on a liquid-fluidized bed [36]. Presumably mesoscale structures affect the flow characteristics profoundly (like is the case in riser flows [38]), and this would provide a stabilizing action against the growth of macroscopic bubbles. To our knowledge we are the first workers able to succeed in fluidizing particles small enough to see an extended interval of uniform expansion with hydrodynamic stabilization. The complexities of hydrodynamic interactions of the pseudoturbulent two-phase flow make a theoretical model capable of predicting the scaling of the solid phase pressure a rather complicated challenge that remains to be addressed.

\section{ACKNOWLEDGMENTS}

We are glad to acknowledge R. Jackson for suggesting to us the reported experiments. This research has been supported by the Xerox Foundation, Spanish Government Agency Ministerio de Ciencia y Tecnologia (DGES) under Contract No. BMF2000-1056, and NATO Grant No. LINKAGE PST.CLG.976575.
[1] D. Geldart, Powder Technol. 7, 285 (1973).

[2] J. Li and J.A.M. Kuipers, Powder Technol. 127, 173 (2002).

[3] J.M. Valverde, M.A.S. Quintanilla, A. Castellanos, and P. Mills, Phys. Rev. E 67, 016303 (2003).

[4] K. Rietema, E.J.E. Cottaar, and H.W. Piepers, Chem. Eng. Sci. 48, 1687 (1993).

[5] S.C. Tsinontides and R. Jackson, J. Fluid Mech. 255, 237 (1993).

[6] N. Menon and D.J. Durian, Phys. Rev. Lett. 79, 3407 (1997).
[7] D.L. Koch and A.S. Sangani, J. Fluid Mech. 400, 229 (1999).

[8] G.D. Cody, D.J. Goldfarb, G.V. Storch, Jr., and A.N. Norris, Powder Technol. 87, 211 (1996).

[9] J.M. Valverde, A. Castellanos, and M.A.S. Quintanilla, Phys. Rev. Lett. 86, 3020 (2001).

[10] M.L. Cowan, J.H. Page, and D.A. Weitz, Phys. Rev. Lett. 85, 453 (2000).

[11] P.N. Segre, F. Liu, P. Umbanhowar, and D.A. Weitz, Nature (London) 409, 594 (2001); P.N. Segre, E. Herbolzheimer, and 
P.M. Chaikin, Phys. Rev. Lett. 79, 2574 (1997).

[12] P. Duru and E. Guazelli, J. Fluid Mech. 470, 359 (2002).

[13] G.K. Batchelor, J. Fluid Mech. 193, 75 (1988).

[14] M. Horio, M.J. Rhodes, X. Wang, M. Nguyen, P. Stewart, and K. Liman, Chem. Eng. Sci. 56, 4433 (2001).

[15] A. Castellanos, J.M. Valverde, and M.A.S. Quintanilla, Phys. Rev. E 64, 041304 (2001).

[16] E. Marring, A.C. Hoffman, and L.P.B.M. Janssen, Powder Technol. 79, 1 (1994).

[17] R. Chirone, L. Massimilla, and S. Russo, Chem. Eng. Sci. 48, 1 (1993).

[18] P. Lettieri, J.G. Yates, and D. Newton, Powder Technol. 110, 117 (2000).

[19] S.C. Saxena and W.Y. Wu, Can. J. Chem. Eng. 77, 312 (1999).

[20] R. Jackson (private communication).

[21] P.K. Watson, J.M. Valverde, and A. Castellanos, Powder Technol. 115, 44 (2001); J.M. Valverde, A. Castellanos, and P.K. Watson, ibid. 118, 240 (2001).

[22] M.A.S. Quintanilla, A. Castellanos, and J.M. Valverde, Phys. Rev. E 64, 031301 (2001).

[23] J.M. Valverde, M.A.S. Quintanilla, A. Castellanos, and P. Mills, Europhys. Lett. 54, 329 (2001).

[24] J.M. Valverde, A. Castellanos, A. Ramos, and P.K. Watson, Powder Technol. 97, 237 (1998).

[25] L. Massimilla and G. Donsi, Powder Technol. 15, 253 (1976); T.G. Mason, A.J. Levine, D. Ertas, and T.C. Halsey, Phys. Rev. E 60, 5044 (2000).
[26] H. Krupp, Adv. Colloid Interface Sci. 1, 111 (1967).

[27] S.T. Nase, W.L. Vargas, A.A. Abatan, and J.J. McCarthy, Powder Technol. 116, 214 (2001).

[28] M.J. Rhodes, X.S. Wang, M. Nguyen, P. Stewart, and K. Liffman, Chem. Eng. Sci. 56, 69 (2001).

[29] R.H. Wilhelm and M. Kwauk, Chem. Eng. Prog. 44, 201 (1948).

[30] G.M. Homsy, Appl. Sci. Res. 58, 251 (1998).

[31] P.U. Foscolo and L.G. Gibilaro, Chem. Eng. Sci. 39, 1667 (1984).

[32] J.F. Richardson and W.N. Zaki, Trans. Inst. Chem. Eng. 32, 35 (1954).

[33] P. Snabre and P. Mills, Eur. Phys. J. E 1, 105 (2000).

[34] J. Verloop and P.M. Heertjes, Chem. Eng. Sci. 25, 825 (1970).

[35] R. Jackson, The Dynamics of Fluidized Particles (Cambridge University Press, Cambridge, 2000).

[36] R. Zenit, M.L. Hunt, and C.E. Brennen, J. Fluid Mech. 353, 261 (1997).

[37] P.U. Foscolo and L.G. Gibilaro, Chem. Eng. Sci. 42, 1489 (1987); L.G. Gibilaro, R. Di Felice, and P.U. Foscolo, Powder Technol. 56, 21 (1988); S. Rapagna, P.U. Foscolo, and L.G. Gibilaro, Int. J. Multiphase Flow 20, 305 (1994); P. Lettieri, S. Brandani, J.G. Yates, and D. Newton, Chem. Eng. Sci. 56, 5401 (2001).

[38] K. Agrawal, P.N. Loezos, M. Syamlal, and S. Sundaresan, J. Fluid Mech. 445, 151 (2001).

[39] S. Sundaresan, Annu. Rev. Fluid. Mech. 35, 63 (2003). 